\title{
Comparison and Polar Opposition
}

\author{
Christopher Kennedy \\ University of California, Santa Cruz
}

\section{Introduction}

The focus of this paper is a phenomenon that I will refer to as "cross-polar anomaly", which is exemplified by the sentences in (1)-(5).1

(1) \#Mike is shorter than Carmen is tall.

(2) \#The Brothers Karamazov is longer than The Idiot is short.

(3) \#The ficus is taller than the ceiling is low.

(4) \#The Tenderloin is dirtier than Pacific Heights is clean.

(5) \# A Volvo is safer than a Fiat is dangerous.

These sentences present an interesting puzzle for a theory of adjectival polarity and the semantics of the gradable adjectives, because they show that comparatives formed out of positive and negative pairs of adjectives are semantically anomalous. Crucially, (1)-(5) clearly contrast with examples of comparative subdeletion in which both adjectives have the same polarity, such as (6) and (7).

(6) The space telescope is longer than it is wide.

(7) The ficus is shorter than the doorway is low, so it should fit in the room.

My goal in this paper is to use cross-polar anomaly as an empirical basis for motivating and developing a particular approach to the semantics of gradable adjectives and the representation of adjectival polarity. Specifically, I will argue that gradable adjectives denote relations between individuals and extents, or intervals on a scale, as in the work of Seuren 1978, 1984, von Stechow 1984b, and Löbner 1990, rather than relations between individuals and degrees, or points on a scale, as traditionally assumed. I will further claim that adjectival polarity should be characterized as a sortal distinction between positive and negative adjectives, I show that this approach supports an explanation of cross-polar anomaly as a type of sortal anomaly.

\section{Degrees and Polar Opposition}

Cresswell (1976:266) suggests that "[w]hen we make comparisons we have in mind points on a scale". Building on this intuition, Cresswell develops a semantic analysis of gradable adjectives as expressions that define mappings between objects and such points, or "degrees". Intuitively, a scale is an abstract representation of measurement: an infinitely long measuring stick, which provides a representation

(C) 1997 by Christopher Kennedy Aaron Lawson (ed), SALT VII, 240-257, Ithaca, NY: Cornell University. 
of the amount to which an object possesses some gradable property. More formally, a scale can be defined as a dense, linearly ordered set of points, where the ordering is relativized to a dimension, which corresponds to a gradable property such as height, length, speed, density, beauty, etc., and a degree can be defined as an element of a scale. ${ }^{2}$

Once scales and degrees are introduced into the ontology, gradable adjectives can be analyzed as relational expressions; specifically, as expressions that relate objects in their domains to degrees on a scale (see e.g. Cresswell 1976, Hellan 1981, Hoeksema 1983, von Stechow 1984a, Heim 1985, Bierwisch 1989, Lerner and Pinkal 1992, 1995, Moltmann 1992, Rullmann 1995, Gawron 1995 and others). ${ }^{3}$ The logical representation of a sentence of the form $x$ is $\varphi$, where $\varphi$ is a gradable adjective, is as shown in (8). (8) has the truth conditions in (9), where $\mathbf{d}_{\varphi}$ is a function that maps objects in the domain of $\varphi$ to the scale associated with $\varphi$.

(8) $\varphi(x, d)$

(9) $\quad\|\varphi(x, d)\|=1$ iff $\mathbf{d}_{\varphi}(x) \geq d$

Informally, $x$ is $\varphi$ is true just in case the projection of $x$ on the scale associated with $\varphi$ (i.e., the degree to which $x$ is $\varphi$ ) is at least as great as some degree $d$. In the case of a typical absolute construction, such as (10), the value of $d$ is a contextually determined "standard" (cf. Bartsch and Vennemann 1973, Cresswell 1976, von Stechow 1984a, Bierwisch 1989, Gawron 1995). 4 For example, the logical representation of a sentence like (10) is (11), where $d_{s(l o n g)}$ denotes a contextually determined standard of "longness".

(10) The Brothers Karamazov is long.

(11) long $\left(B K, d_{s(l o n g)}\right)$

Given the truth conditions in (9), (10) is true if and only if $\mathbf{d}_{\text {long }}(B K) \geq d_{s(\text { long) }}$ holds, i.e., just in case the projection of The Brothers Karamazov on a scale of length is at least as great as the standard of longness in the context of utterance.

Absolute constructions with negative adjectives can be analyzed in basically the same way, with one important modification: the relation between the reference value and the standard value must be reversed (see Bierwisch 1989, Gawron 1995 for discussion). This point is illustrated by the analysis of (12).

(12) The Dream of a Ridiculous Man is short.

(13) $\operatorname{short}\left(\right.$ Dream, $\left.d_{s(\text { short })}\right)$

The logical representation of (12) is (13), where $d_{s(s h o r t)}$ denotes an appropriate standard of "shortness" (which may or may not be the same as the standard of longness in a given context; see Klein 1980). The crucial difference between (12) and (10) is that in the former, the partial ordering relation associated with the absolute construction must be reversed: (12) is true just in case the degree to which The Dream of a Ridiculous Man is short is ordered below the standard value on a 
scale of length. To see that this is so, consider a context in which the standard of longness for Russian novels is 600 pages, and the standard of shortness is 400 pages. In this context, any novel over 600 pages in length counts as long, while any novel under 400 pages in length counts as short (a novel whose length is between 400 and 600 pages is neither long nor short; in Klein's (1980) terminology, it falls in the "extension gap" on the scale of length).

The analysis of the absolute constructions outlined here can be used as the basis for an analysis of comparatives as expressions that introduce existential quantification over degrees (see e.g., Hellan 1981, von Stechow 1984a,b, Heim 1985, Lerner \& Pinkal 1992, 1995, Gawron 1995, Hazout 1995, Rullmann 1995). For example, in Heim 1985, comparatives are analyzed as indefinite degree descriptions, which restrict the possible values of the degree argument of a gradable predicate. 5 On this view, the logical representation of a typical comparative like (14) is as shown in (15), where $d_{c}$ is the degree introduced by the comparative clause (the complement of than or as). 6

(14) $x$ is more $\varphi$ than $d_{c}$

(15) $\exists d\left[d>d_{c}\right][\varphi(x, d)]$

Given the truth conditions for the absolute construction in (9) above, $x$ is more $\varphi$ than $d_{c}$ is true just in case there is a degree $d$ such that $d$ exceeds $d_{c}$ and $x$ is at least as $\varphi$ as $d$. Equatives and less comparatives are interpreted in the same way, modulo the different ordering relations introduced by as and less ( $\geq$ and $<$, respectively).

For illustration of the basic proposal, consider the analysis of (16), which has the logical representation in (17).

(16) The Brothers Karamazov is longer than The Idiot is.

(17) $\exists d\left[d>1 d^{\prime}\right.$.long $\left(\right.$ Idiot,$\left.\left.d^{\prime}\right)\right][$ long $(B K, d)]$

(16) is true iff there is a degree such that $d$ exceeds the (maximal) degree to which The Idiot is long, and The Brothers $K$. is at least as long as $d$. In a context such as (18), then, where $d_{I}$ denotes the degree of The Idiot's length and $d_{B K}$ denotes the degree of The Brothers Karamazov's length, (16) is true.

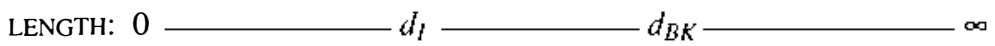

Comparatives with negative adjectives can be analyzed in essentially the same way, with one exception: as was the case with negative absolute constructions, the ordering relation associated with the comparative must be reversed. This is illustrated by a sentence like (19), which has the logical representation in (20).

(19) The Idiot is shorter than The Brothers Karamazov is.

(20) $\exists d\left[d<1 d^{\prime} . \operatorname{short}\left(B K, d^{\prime}\right)\right][\operatorname{short}($ Idiot,$d)]$ 
(19) is true just in case for some degree $d$ which is exceeded by the degree to which The Brothers Karamazov is short, The Idiot is $d$-short. It follows that this sentence is also true in the context illustrated in (18), which is the correct result.

The relational difference between positive and negative adjectives illustrated by the absolute and comparative constructions discussed here suggests an intuitive approach to the representation of adjectival polarity within a degree algebra. Specifically, we can make the natural assumption that antonymous adjectives such as long and short map objects in their domain to the same points on a shared scale-in the case of long and short, a scale along a dimension of length-but they are distinguished by having opposite ordering relations imposed on the degrees (cf. Rullmann 1995, Kennedy 1997). In other words, the sets of positive and negative degrees on any scale are isomorphic, but they stand in the dual relation to each other. This assumption has a very positive result: if for any object, its degrees of e.g. longness and shortness are the same, then sentences like (16) and (19) are logically equivalent. As a result, $(21)$ is correctly predicted to be valid.

(21) The Brothers Karamazov is longer than The Idiot if and only if The Idiot is shorter than The Brothers Karamazov.

\section{The Problem of Cross-Polar Anomaly}

In its most general sense, the puzzle presented by sentences like (1)-(5) for the analysis of gradable adjectives and adjectival polarity outlined in section 2 is that the very same assumptions which lead to a straightforward explanation of the validity of (21) make the wrong predictions in the case of cross-polar anomaly. Consider (2) again, repeated below.

(2) \#The Brothers Karamazov is longer than The Idiot is short.

(22) $\exists d\left[d>d^{\prime}\right.$. short $\left(\right.$ Idiot,$\left.\left.d^{\prime}\right)\right][\operatorname{long}(B K, d)]$

(1) has the logical representation shown in (22): it's true just in case for some degree $d$ which exceeds the (maximal) degree to which The Idiot is short, The Brothers Karamazov is at least as long as $d$. Since the degree of an object's shortness is identical to its degree of longness, this sentence should be true whenever the projection of The Brothers Karamazov on a scale of length exceeds the projection of The Idiot on a scale of length. In the context illustrated above in (18), then, (2) should be true. In other words, (2) should not only be perfectly interpretable, it should be logically equivalent to (16) and (19). Crucially, this result is not specific to long and short: it is inevitable given the formalization of degrees as points on a scale and the assumption that positive and negative degrees are the same objects, an assumption that is necessary to explain the validity of (21).

Note that this result is not a consequence of the assumption that the comparative clause denotes a definite description of a (maximal) degree. The same problem arises if the comparative clause is analyzed as a universal quantification 
structure (as in e.g., Atlas 1984, Lerner \& Pinkal 1992, 1995, Gawron 1995, Moltmann 1992, Gawron 1995), although such an analysis appears at first glance to provide an explanation of cross-polar anomaly. If the comparative clause involves universal quantification, the logical form of (2) is (23).

$$
\exists d\left[\forall d^{\prime}\left[\operatorname{short}\left(\operatorname{Idiot}, d^{\prime}\right) \rightarrow d>d^{\prime}\right]\right][\operatorname{long}(B K, d)]
$$

Given the earlier claim that the truth conditions of the absolute are defined in terms of a partial ordering relation (i.e., that $\varphi(x, d)$ is true iff $x$ is at least as $\varphi$ as $d$ ), (2) is true just in case for some degree $d$ that exceeds every degree $d^{\prime}$ such that The Idiot is at least as short as $d^{\prime}$, The Brothers $K$ is at least as long as $d$. Assuming that a scale has no maximal element (see von Stechow 1984a,b, Rullmann 1995), however, there is no degree that satisfies the conditions imposed by the restriction, because the set of degrees introduced by the comparative clause includes every degree ordered above the projection of The Idiot on a scale of length. The result is that sentences like (2) are predicted to be contradictory.

Even if we are willing to explain cross-polar anomaly in terms of contradiction, however, there is evidence that this analysis cannot be maintained. Comparatives with less show that if the comparative clause involves universal quantification over degrees, then the truth conditions of the absolute cannot be defined in terms of a partial ordering relation (see Rullmann 1995). Consider, for example, the analysis of (24).

(24) The Devils is less acclaimed than The Brothers Karamazov is.

(25) $\exists d\left[\forall d^{\prime}\left[\right.\right.$ acclaimed $\left.\left.\left(B K, d^{\prime}\right) \rightarrow d<d^{\prime}\right]\right][$ acclaimed $($ Devils, $d)]$

If the set of degrees introduced by the comparative clause includes every degree that is at least as great as the degree to which The Brothers Karamazov is acclaimed, then there is no degree that satisfies the restriction, incorrectly predicting that (24) should be contradictory. In order to resolve this problem, the truth conditions of the absolute must be defined in terms of equality: $\varphi(x, d)$ is true iff $x$ is exactly as $\varphi$ as $d$ ). The positive consequence of this move is that the set of degrees introduced by the comparative clause in (25) contains a single member-the degree to which The Brothers Karamazov is acclaimed-permitting an accurate analysis of the truth conditions of comparatives with less. The negative consequence is that the set of degrees introduced by the comparative clause in (23), also contains a single member-the degree to which The Idiot is short-with the result that (2) (as well as other examples of cross-polar anomaly) is no longer predicted to be contradictory.

A more promising response to the puzzle of cross-polar anomaly would be to reject the claim that positive and negative degrees are comparable in the way that I have assumed, arguing instead that cross-polar anomaly is an instance of incommensurability, a type of anomaly observed in comparatives constructed out of incomparable adjectives, such as (26)-(27) (see Klein 1991 for discussion).

\#Carmen is taller than Mike is intelligent. 
\#The Idiot is more tragic than my copy of The Brothers $K$ is heavy.

The formalization of scales and degrees outlined in section 2 provides the basis for an explanation of incommensurability as a kind of sortal anomaly. Scales are associated with a dimensional parameter, which differentiates one scale from another, and has the consequence that degrees on different scales are elements of different ordered sets. Since the ordering relation introduced by the comparative is defined only for elements of the same ordered set, in an example like (27), which has the logical representation in (28), only degrees of heaviness satisfy the restriction imposed by the comparative.

\section{$\exists d\left[d>\mathrm{l} d^{\prime} . h e a v y\left(\right.\right.$ my copy of the Brothers $\left.\left.K, d^{\prime}\right)\right][$ tragic(Idiot, $\left.d)\right]$}

As a result, the degree argument of tragic is constrained to be a degree of heaviness. Degrees on scales associated with different dimensional parameters are distinct objects, however, so the comparative restricts the degree argument of tragic to be a degree of the wrong sort, triggering a sortal anomaly. ${ }^{7}$

This explanation could be extended to cross-polar anomaly in the following way. Like degrees of e.g. tragicness and heaviness, positive and negative degrees on the same scale are different sorts of objects, and sentences like (1)-(5) are anomalous for the same reason as (26)-(27): the comparative restricts the degree argument of the adjective to be a degree of the wrong sort. This explanation builds on the intuition that although positive and negative adjectives share the same scale (an assumption that is necessary to account for the validity of e.g. (21)), they provide different perspectives on the projection of an object onto the scale. As a result, positive and negative degrees should be treated as different sorts of objects.

Although I will ultimately argue that the basic form of this explanation of cross-polar anomaly is the right one, this analysis is unavailable in a degree algebra for two reasons. First, typical examples of comparative subdeletion such as (6)-(7) show that whenever a mapping between degrees on different scales can be defined, comparison is possible. Given the analysis of adjectival polarity outlined in section 2 , such a mapping between positive and negative degrees is always available (the identity function), therefore examples of cross-polar anomaly should be interpretable. Second, and more problematic, if positive and negative degrees are the same objects, then they simply can't be sortally distinguished (in the way that degrees of tragicness and heaviness can be) without resorting to ad hoc stipulations.

\section{Extents and Polar Opposition}

As noted above, the intuition underlying an explanation of cross-polar anomaly in terms of incommensurability is that although antonymous pairs of adjectives provide the same kind of information about an object-both tall and short are used to characterize an object's height-they nevertheless represent different perspectives on the projection of an object on a scale, and this difference in perspective should be 
formalized in terms of a sortal distinction between positive and negative degrees. The problem for a degree algebra is that such a distinction must be stipulated, it does not follow from the underlying architecture of the theory of adjectival polarity.

In order to construct a theory of polarity that does have this property, I will adopt an alternative ontology of "degrees", in which the projection of an object on a scale is represented not as a discrete point, but rather as an interval, or extent (see Seuren 1978, 1984, von Stechow 1984b, and Löbner 1990). 8 This idea can be formalized as follows. As in the degree approach, a scale can be defined as a dense, linearly ordered set of points possibly with a minimal element but with no maximal element, where the ordering is relative to some dimension. ${ }^{9}$ An extent can then be defined as a convex, proper subset of a scale. Finally, assuming a function d which maps an object in the domain of a gradable adjective to a point on the scale (this is the same sort of function that was adopted earlier to map objects to degrees), I will make a further distinction between two sorts of extents which, following von Stechow 1984b, I will refer to as positive and negative extents. Positive and negative extents are defined in (29) and (30).

(29) The positive extent of $a$ on a scale $S_{\delta}\left(\operatorname{POS}_{\delta}(a)\right)=\left\{p \in S_{\delta} \mid p \leq \mathbf{d}_{\delta}(a)\right\}$

(30) The negative extent of $a$ on a scale $S_{\delta}\left(\mathrm{NEG}_{\delta}(a)\right)=\left\{p \in S_{\delta} \mid \mathbf{d}_{\delta}(a) \leq p\right\}$

Roughly speaking, a positive extent is an extent which ranges from the lower end of a scale to a some point, and a negative extent is an extent which ranges from some point to the upper end of the scale. The basic idea is illustrated by (31), which shows the positive and negative projections of an object $a$ on a scale $S_{\delta}$.

$$
S_{\delta}: \quad 0 \ldots \operatorname{POS} \delta(a) \longrightarrow \mathrm{NEG}_{\delta}(a) \longrightarrow \infty
$$

The intuition that this distinction between positive and negative extents is designed to capture is exactly the one I discussed above: that the members of an antonymous pair of adjectives provide different-in fact complementary-perspectives on the projection of an object onto the scale. The structural distinction between positive and negative extents provides a means of explicitly encoding this difference into a theory of adjectival polarity. Because positive and negative extents are distinct objects, the set of positive extents on a scale $S$ and the set of negative extents on $S$ are disjoint subsets of the entire set of extents on $S$. As a result, the set of gradable adjectives can be sorted in the following way: positive adjectives denote relations between individuals and positive extents; negative adjectives denote relations between individuals and negative extents. On this view, adjectival polarity is characterized as a sortal distinction between positive and negative adjectives.

This approach to adjectival polarity has an important empirical consequence: it allows the interpretation of absolute and comparative constructions to be defined in terms of a single ordering relation. 10 Since extents are defined set-theoretically, the truth conditions for both positive and negative adjectives in the absolute form can be stated in terms of set-inclusion, as in (32), where $\Phi$ is a function from individuals to positive or negative extents (determined by the polarity of $\varphi$ ). 


$$
\|\varphi(x, e)\|=1 \text { iff } \Phi(x) \supseteq e
$$

For illustration, consider the analysis of (33) and (34) in the context illustrated by (35), where $\operatorname{LONG}(B K)$ denotes the positive extent of The Brothers Karamazov on the scale of length, SHORT $(B K)$ denotes its negative extent, and $e_{s(\text { long })}$ and $e_{s(\text { short })}$ denote appropriate standards of longness and shortness, respectively.

$$
\begin{array}{lll}
\text { (33) The Brothers Karamazov is long. } & \Rightarrow & \operatorname{long}\left(B K, e_{\text {sf }} \text { (eng }\right) \\
\text { (34) The Brothers Karamazov is short. } & \Rightarrow & \operatorname{short}\left(B K, e_{\text {sf short })}\right)
\end{array}
$$

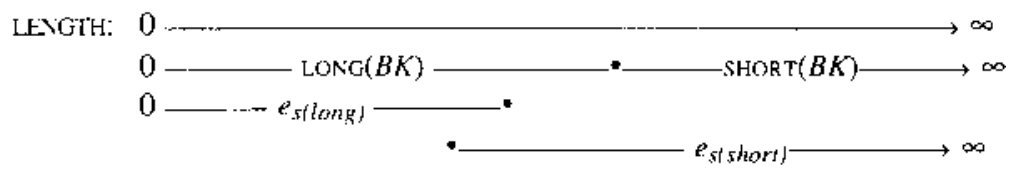

According to the truth conditions for the absolute in (32), (33) is true just in case the extent to which The Brothers Karamazov is long includes the standard value. It follows that (33) is true in (35), because $\operatorname{LONG}(B K) \supseteq e_{s(l o n g)}$ holds.

The negative absolute constructions in (34) is analyzed in exactly the same way. (34) is true just in case the extent to which The Brothers Karamazov is short includes the standard of shortness; in the context illustrated by (35), (34) is false, because $\operatorname{SHORT}(B K) \supseteq e_{s(\text { short })}$ does not hold. The same ordering relation is used to calculate the truth of both the positive and negative absolute constructions; this was not the case in the algebra of degrees discussed in section 2 , in which the ordering relation associated with the absolute had to be reversed for negative adjectives.

The analysis of positive and negative comparatives is similar. To keep the comparison as simple and direct as possible, I will adopt the same kind of semantic analysis of comparatives that I used when describing the degree approach: comparatives involve restricted existential quantification over extents, and the comparative clause is a definite description of an extent. Given this assumption, the interpretation of the comparative construction can be stated in terms of proper inclusion: a sentence of the form $x$ is more $\varphi$ than $d_{c}$ has the interpretation in (36).

$$
\exists e\left[e \supset e_{c}\right][\varphi(x, e)]
$$

For illustration, consider the analysis of (37) and (38) in the context represented by (39), where LONG(BK) and SHORT(BK) are as defined above, and LONG(Idiot) and SHORT(Idiot) represent the positive and negative extents of The Idiot's length.

(37) The Brothers Karamazov is longer than The Idiot is.

(38) The Idiot is shorter than The Brothers Karamazov is.

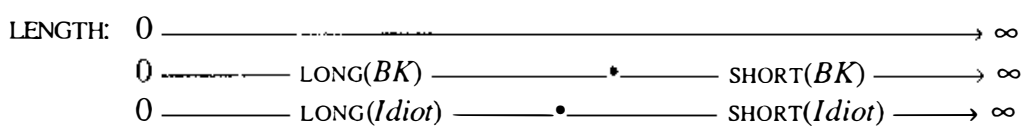


The logical representation of (37) is (40): this sentence is true just in case for some extent $e$ such that $e$ properly includes the positive projection of The Idiot on a scale of length, The Brothers Karamazov is at least as long as $e$.

$$
\exists e\left[e \supset \mathfrak{l} e^{\prime} . \operatorname{long}\left(\text { Idiot }, e^{\prime}\right)\right][\operatorname{long}(B K, e)]
$$

Since these conditions are met in the context shown in (39), (37) is true.

The negative comparative (38) is treated in the same way, and like (37), it is true in (39). The logical representation of (38) is (41), which states that (38) is true just in case for some extent $e$ such that $e$ properly includes the negative projection of The Brothers $K$ on a scale of length, The Idiot is at least as short as $e$.

\section{(41) $\exists e\left[e \supset e^{\prime} \cdot \operatorname{short}\left(B K, e^{\prime}\right)\right][\operatorname{short}($ Idiot,$e)]$}

Note that as was the case with the negative absolute, there is no need to assume a change in the ordering relation associated with the comparative: the structure of extents is such that the same ordering relation can be used to characterize the truth conditions of both positive and negative comparatives.

(37) and (38) can be used to illustrate another important aspect of the analysis, namely that it explains the validity of constructions like (42).

(42) The Idiot is shorter than The Brothers Karamazov iff The Brothers Karamazov is longer than The Idiot.

A sentence like (42) can be paraphrased in the following way: "the extent to which The Idiot is short exceeds the extent to which The Brothers Karamazov is short if and only if the extent to which The Brothers Karamazov is long exceeds the extent to which The Idiot is long". More generally, statements like (42) can be viewed as substitution instances of (43), where $\operatorname{POS}_{\delta}$ and $\mathrm{NEG}_{\delta}$ are antonymous gradable adjectives whose ranges are extents on a scale associated with a dimension $\delta$.

$$
\operatorname{POS}_{\delta}(a) \supset \operatorname{POS}_{\delta}(b) \text { iff } \mathrm{NEG}_{\delta}(b) \supset \operatorname{NEG}_{\delta}(a)
$$

The validity of (43) follows from the fact that positive and negative extents represent (join) complementary projections of an object on a scale. Given the definitions in (29) and (30), the complements of positive and negative extents are:

$$
\begin{aligned}
& \text { (44) }-\mathrm{NEG}_{\delta}(x)=\operatorname{POS}_{\delta}(x)-\left\{\mathbf{d}_{\delta}(x)\right\} \\
& (45)-\operatorname{POS}_{\delta}(x)=\operatorname{NEG}_{\delta}(x)-\left\{\mathbf{d}_{\delta}(x)\right\}
\end{aligned}
$$

Returning to (43), if $\operatorname{POS}_{\delta}(a) \supset \operatorname{POS}_{\delta}(b)$, then $\operatorname{POS}_{\delta}(a)-\left\{\mathbf{d}_{\delta}(a)\right\} \supset \operatorname{POS}_{\delta}(b)-\left\{\mathbf{d}_{\delta}(b)\right\}$, since $\mathbf{d}_{\delta}(a)$ and $\mathbf{d}_{\delta}(b)$ are the maximal elements of $\operatorname{POS}_{\delta}(a)$ and $\operatorname{POS}_{\delta}(b)$, respectively. It follows that $-\mathrm{NEG}_{\delta}(a) \supset-\mathrm{NEG}_{\delta}(b)$, by substitution, and finally that $\mathrm{NEG}_{\delta}(b) \supset \operatorname{NEG}_{\delta}(a)$, by contraposition. The other direction of the biconditional can be proved in the same way. 


\section{Cross-Polar Anomaly Explained}

We are now in a position to see how the algebra of extents and the sortal characterization of adjectival polarity supports an explanation of cross-polar anomaly. A consequence of the semantic analysis of comparatives presented in section 4 is that the polarity of the adjective in the comparative clause determines the sortal value of the extent variable that the comparative quantifies over. To see why, consider the logical representation of the comparative, repeated in (46).

$$
\exists e\left[e \supset e_{c}\right][\varphi(x, e)]
$$

When the extent introduced by the comparative clause $\left(e_{c}\right)$ is positive, it is an interval that ranges from the lower end of the scale to some point. In order for a proper inclusion relation to hold between $e$ and $e_{c}, e$ must also include the lower end of the scale, therefore $e$ must also be positive. When $e_{c}$ is negative, it ranges from the upper end of the scale to some point. It follows that in order for $e$ to properly include $e_{c}$, it also must be negative, since it must include the upper end of the scale. The result is that a comparative restricts the possible value of the extent variable it binds to be an extent of the same sort as that selected by the adjective in the comparative clause. This entails that whenever the adjective in the comparative clause is of opposite polarity from the adjective in the main clause, the comparative restricts the extent argument of the main adjective to be an extent of the wrong sort. This sortal mismatch is the cause of cross-polar anomaly.

For illustration of the analysis, consider sentences (1) and (2). The logical form of (1), in which the adjective in the main clause is negative and the adjective in the comparative clause is positive, is (47).

(1) \#Mike is shorter than Carmen is tall.

(47) $\exists e\left[e \supset e^{\prime} . t a l l\left(C a r m e n, e^{\prime}\right)\right][\operatorname{short}($ Mike,e $)]$

The comparative restricts the possible value of the extent argument of short to be an extent which properly includes the extent to which Mike is tall. Only positive extents satisfy the restriction, therefore the comparative restricts the extent argument of the negative adjective short to be a positive extent, triggering sortal anomaly.

The anomaly of (2), in which the adjectives are reversed, is explained in exactly the same way. (2) has the logical representation in (48).

(2) \#The Brothers Karamazov is longer than The Idiot is short.

(48) $\exists e\left[e \supset e^{\prime} . \operatorname{short}\left(\right.\right.$ Idiot,$\left.\left.e^{\prime}\right)\right][\operatorname{long}(B K, e)]$

According to (48), (2) is true iff for some extent $e$ that properly includes the extent to which The Idiot is short, The Brothers $K$ is at least as short as $e$. Only negative extents satisfy the restriction imposed by the comparative, so the extent argument of long is constrained to be a negative extent, again resulting in sortal anomaly.

The basic form of this explanation is the same as the one I rejected in my 
earlier discussion of the degree approach in section 3: sentences like (1)-(5) are anomalous because positive and negative adjectives are incommensurable. This type of analysis is available in the extent approach because the algebra of extents and the characterization adjectival polarity presented in section 4 makes a basic, structural distinction between positive and negative extents: positive and negative extents, unlike positive and negative degrees, are different sorts of objects. As a result, when a comparative restricts the argument of the adjective it modifies to be an extent of the wrong polarity, it triggers a sortal anomaly. It should be noted that this "polar incommensurability" is different in an important way from the "dimensional incommensurability" discussed in section 3, which involved adjectives on different scales (see (26) and (27)). Whereas the latter is triggered when a gradable adjective is supplied with an extent argument from an incompatible scale, the former arises when an adjective is supplied with an extent on the correct scale, but not "of the right shape".

An interesting consequence of this analysis is that cross-polar anomaly forms a natural class with another well known class of anomalous sentences: examples in which a negative adjective is associated with a measure phrase, as in (49) (see Hale 1970 for early discussion of this phenomenon).

\#The Dream of a Ridiculous Man is 21 pages short.

The explanation for the anomaly of (49), which is roughly the same as the one suggested in von Stechow 1984b, runs as follows. Positive extents on a scale with a zero point-which is the type of scale I assume to be associated with dimensional adjectives like long and short-are bounded. In contrast, negative extents are always unbounded, since a scale has no maximal element. Assuming that measure phrases like 21 pages, 2 meters, and so on, denote bounded extents, the measure phrase in a sentence like (49) provides the wrong sort of argument for the negative adjective short. The end result is that (49), like cross-polar anomaly, is an example of "polar incommensurability".

\section{Positives That Look Like Negatives}

In the remaining sections of this paper, I will take a quick look at two sets of facts which, on the surface, appear to be counterexamples to the claim that comparatives constructed out of positive and negative pairs of adjectives are anomalous. The first set of facts, brought to my attention by Chris Barker (personal communication), is illustrated by the sentences in (50)-(52).

(50) Your $\mathrm{C}$ is sharper than your $\mathrm{D}$ is flat.

(51) My watch is faster than your watch is slow.

(52) She was earlier than I was late.

These sentences are clearly not anomalous, but there is good reason to believe that 
this is not because the analysis developed in the previous section is incorrect. Instead, despite initial appearances, all of the adjectives in (50)-(52) are positive. Three pieces of evidence support this conclusion.

First, all of the adjectives in (50)-(52) can take measure phrases; as observed in section 5 , this is a characteristic of positive adjectives, not negatives.

(53) Your C is $30 \mathrm{~Hz}$ flat/sharp.

(54) My watch is 10 minutes fast/slow.

(55) She was an hour early/late.

Second, (50)-(52) are non-anomalous only on a very specific interpretation: one in which the extents involved in the interpretations of the adjectives measure divergence from some common point of reference, rather than the "absolute" projection of an object on a scale. For example, (50) compares the extents two which two notes differ from their respective "pure tones", and (51) compares the extents to which our watches deviate from "on time". When absolute extents are compared, as in typical cases of cross-polar anomaly, the adjectives in (50)-(52) trigger cross-polar anomaly. This is shown by the minimal pair (51) and (56).

\section{(56) \#My car is faster than your car is slow.}

Finally, when the adjectives in (50)-(52) are interpreted in as described above, statements that appear to be substitution instances of (43) are not valid. (57), for example, is false when your $C$ is sharper than your $D$, but neither is flat.

Your $\mathrm{C}$ is sharper than your $\mathrm{D}$ iff your $\mathrm{D}$ is flatter than your $\mathrm{C}$.

If the adjectives in (50)-(52) are sortally positive, as these facts suggest, then they do not represent counterexamples to the analysis of cross-polar anomaly proposed in the previous section. An important question that remains to be answered, however, is how these adjectives come to have the interpretations they do-how is it, for example, that fast and slow can either have canonical polar interpretations, as in (56), or the"measuring from a reference point" interpretation in (51)? This is a question that should be a point of future work, but I will not attempt to answer it here.

\section{Comparison of Deviation}

The second set of facts that appear to be counterexamples to the generalization that comparatives constructed out of antonymous adjectives are anomalous is exemplified by the equatives (58) and (59) and the paratactic comparatives (60) and (61).

(58) Shawn is as tall as Mugsy is short.

(59) The Tenderloin is as dirty as Pacific Heights is clean. 
(60) A Fiat is more dangerous than a Volvo is safe.

(61) San Francisco Bay is more shallow than Monterey Bay is deep.

These sentences, which I will refer to as "comparison of deviation" constructions, have three characteristics that distinguish them from standard comparatives.

First, comparison of deviation constructions compare the relative extents to which two objects deviate from some standard value associated with the adjective. This is illustrated by (62), which accurately paraphrases the meaning of (58).

(62) The extent to which Shawn exceeds a standard of tallness is at least as great as the extent to which Mugsy exceeds a standard of shortness.

In contrast, standard comparatives and equatives compare the "absolute" projections of two objects on a scale. A sentence like (63), has the paraphrase in (64).

(63) Shawn's feet are as wide as Mugsy's feet are long.

(64) The extent to which Shawn's feet are wide is at least as great as the extent to which Mugsy's feet are long.

Second, unlike typical comparatives, comparison of deviation constructions entail that the properties predicated of the compared objects are true in the absolute sense. For example, (65) is contradictory, but (66) is not.

(65) San Francisco Bay is more shallow than Monterey Bay is deep, but it's still quite deep.

(66) San Francisco Bay is shallower than Monterey Bay, but it's still quite deep.

Finally, comparison of deviation constructions are morphologically dis-tinguished from typical comparatives in not licensing incorporation of the adjective and comparative morpheme, as shown by the contrasts between (61) and (67).11

(67) \#San Francisco Bay is shallower than Monterey Bay is deep.

These characteristics suggest that despite their superficial similarity to sentences which show cross-polar anomaly, comparison of deviation constructions are semantically-and possibly syntactically-distinct, and so not counterexamples to the analysis developed in section 5. In support of this claim, it should be noted that a sentence like (58) cannot mean that Shawn and Mugsy are equal in height. This is a positive result, because this is exactly the kind of interpretation that the explanation of cross-polar anomaly proposed here rules out. The "standard" logical representation of (58) is (68), which is true just in case there is an extent $e$ such that $e$ includes the extent to which Mugsy is short, and Shawn is at least as tall as $e$.

$$
\exists e\left[e \supseteq e^{\prime} . \operatorname{short}\left(\text { Mugsy, } e^{\prime}\right)\right][\operatorname{tall}(\text { Shawn,e })]
$$


The interpretation of (58) indicated by (68) is impossible because it triggers crosspolar anomaly: the equative restricts the extent argument of the adjective in the main clause to be an extent of the wrong sort.

The question that remains to be answered, though, is just how is the comparison of deviation interpretation of (58) (as well as (59)-(61)) derived? Space prohibits a fully detailed analysis of this phenomenon here, so I will restrict myself to an general overview (see Kennedy 1997 for a detailed analysis of comparison of deviation). The explanation builds on the interpretation of "differential comparatives" like (69)-(70) (see Hellan 1981 and von Stechow 1984a for discussion of differential comparatives).

(69) The Brothers Karamazov is 122 pages longer than The Idiot.

(70) Mike is $12 \mathrm{~cm}$ shorter than Carmen.

The interesting aspect of these constructions is the interpretation of the measure phrases. In (69) and (70), 122 pages and $12 \mathrm{~cm}$ denote "differential extents": extents that describe the difference between the projections of the compared objects on a scale.

What I would like to suggest is that comparison of deviation constructions involve quantification over such differential extents, rather than over the actual extent argument of a gradable predicate, as in standard comparatives. More specifically, comparison of deviation involves quantification over a differential extent that denotes the difference between the absolute projection of an object and a contextually determined standard value. If this analysis is correct, then assuming an operation of extent concatenation as defined in (71) (see von Stechow 1984b, Bierwisch 1989; see also Hellan 1981, von Stechow 1984a, and Klein 1991 for concatenation of degrees), the logical representation of a sentence like (58) can be formalized as in (72): to paraphrase, (58) is true just in case for some extent $e$ such that $e$ is at least as great as the extent to which Mugsy exceeds a standard of shortness, Shawn exceeds a standard of tallness by $e$.

(71) $e_{1} \bullet e_{2}=\imath e\left[e-e_{2}=e_{1}\right]$.

(72) $\exists e\left[e \supseteq e^{\prime} . \operatorname{short}\left(\right.\right.$ Mugsy, $\left.\left.e^{\prime} \bullet e_{s(\text { short })}\right)\right]\left[\operatorname{tall}\left(\operatorname{Shawn}, e^{\bullet} e_{s(\text { tall })}\right)\right]$

This analysis accurately captures the meaning of this sentence, and it also accounts for its entailments: since a sentence of the form $x$ is $\varphi$ is true just in case the extent to which $x$ is $\varphi$ is at least as great as the standard for $\varphi$, the truth conditions for the absolute are satisfied whenever the truth conditions for the comparison of deviation interpretation are satisfied.

More importantly, if this analysis is correct, then it explains why comparison of deviation does not trigger cross-polar anomaly. Simply put, differential extents are sortally the same regardless of the sortal value of the extent with which they are concatenated. Empirically, this is shown by the fact that differential measure phrases occur with both positive and negative comparatives, as illustrated by (70). More generally, this follows from the algebra of extents: in 
order for the concatenation of a differential extent with a positive or negative extent to be an interval that satisfies the definition of extent (a convex proper subset of a scale; see the discussion in section 4), the differential extent must be bounded. Assuming that all bounded extents are sortally the same (see the discussion of (49) above), if comparison of deviation involves quantification over differential extents, it should not trigger cross-polar anomaly. What remains, of course, is to show exactly how comparison of deviation interpretations are compositionally derived.

\section{Conclusion}

Using the phenomenon of cross-polar anomaly as an empirical basis for the argumentation, this paper has made three primary claims. First, the interpretation of gradable adjectives should be formalized in terms of extents, or intervals on a scale, rather than degrees (points on a scale). Second, two sorts of extents should be distinguished-positive extents and negative extents-in such a way that they provide complementary perspectives on the projection of an object on a scale, as argued in Seuren 1978, 1984, von Stechow 1984b, and Löbner 1990. Third, adjectival polarity should be characterized as a sortal distinction: positive adjectives denote relations between individuals and positive extents; negative adjectives denote relations between individuals and negative extents. This set of assumptions supports a general semantic analysis of positive and negative gradable adjectives in both the comparative and absolute forms, and also explains the validity of statements like (42). Most importantly, it provides a principled explanation of cross-polar anomaly as a type of sortal anomaly.

\section{Endnotes}

*I am grateful to Chris Albert, Chris Barker, Donka Farkas, and Bill Ladusaw for extremely valuable discussion of the material presented here and for comments on earlier drafts. Errors or inconsistencies are my responsibility.

1. The anomaly of sentences like (1)-(5) was first observed by Hale 1970. See Bierwisch 1989 for discussion of similar facts in German.

2. The importance of the "dimensional parameter" is that it provides a means of differentiating one scale from another, and by extension, a means of distinguishing e.g. measurements of tallness from measurements of fastness.

3. A different type of analysis with the general framework of scalar approaches, in which adjectives are analyzed as measure functions-functions from objects to scalar values-is developed in Wunderlich 1970, Bartsch and Vennemann 1972, and Kennedy 1997.

4. Intuitively, a standard-denoting degree is a degree that identifies a point on a scale that supports a partitioning of those objects in the domain of a gradable adjective $\varphi$ for which the statement $x$ is $\varphi$ is true, from those objects for which $x$ is $\varphi$ is false, in some context (cf. Klein 1980). In the discussion that follows, I will 
leave open the question of how exactly the standard value is identified, and simply assume (following Bierwisch 1989) that it is determined contextually, relative to some subset of the domain of the gradable adjective determined to be relevant (i.e., a comparison class; cf. the non-scalar approaches to the semantics of gradable adjectives in McConnell-Ginet 1973, Kamp 1975, Klein 1980, Larson 1988).

5. Although many scalar analyses of comparatives are stated in terms of existential quantification over degrees, some analyze comparatives as universal quantification structures (Cresswell 1976; cf. Postal 1974, Williams 1977) or as generalized quantifiers (Moltmann 1992, Hendriks 1995). I focus here on an existential quantification analysis for perspicuity, but the problem of cross-polar anomaly that I will introduce below holds for all accounts in which degrees are analyzed as points on a scale. See Kennedy 1997 for discussion.

6. For simplicity, I assume here that the comparative clause is a definite description of a maximal degree (see Russell 1905, Postal 1974, Williams 1977, Hellan 1981, von Stechow 1984a, Rullmann 1995, Kennedy 1997). However, the problem presented by cross-polar anomaly for a degree-based analysis of gradable adjectives and comparatives extends to analysis in which the comparative clause is analyzed as a universal quantification structure, as in Cresswell 1976, Lerner \& Pinkal 1992, Moltmann 1992, and Gawron 1995, as will be made clear in section 3.

7. To be precise, this sortal anomaly arises because the partial ordering relation associated with the absolute construction is undefined since its arguments (the degree introduced by the comparative and the projection of the subject on a scale of "tragedy") come from different scales.

8. Bierwisch (1989) also constructs an algebra in which degrees are formalized as intervals on a scale, but this algebra does not include analogs to negative extents (see the discussion below). Although I do not have space to go through Bierwisch's proposals in detail here, it should be noted that his assumptions provide a means of ruling out examples of cross-polar anomaly in which the adjective in the comparative clause is negative as semantically anomalous. Sentences in which the adjective in the comparative clause is positive are not semantically anomalous, however, but are instead predicted to be contradictory, suggesting that there should be an observable difference between e.g. (1) and (2). Such a difference does not appear to exist.

9. I assume that dimensional adjectives like long and tall are associated with scales which have minimal elements, though we may not want to make this assumption for adjectives like beautiful or interesting; see Bierwisch 1989 for discussion.

10. In effect, the structural distinction between positive and negative extents derives the relational difference between positive and negative adjectives. See Kennedy 1996, 1997 for additional discussion of this point, and discussion of how the algebra of extents derives the monotonicity properties of gradable adjectives (cf. Seuren 1978, Sánchez-Valencia 1994).

11. I won't have anything to say about these facts here, though they suggest that comparison of deviation constructions are syntactically distinct from standard comparatives. 


\section{References}

Atlas, J. 1984. Comparative adjectives and adverbials of degree: An introduction to radically radical pragmatics. Linguistics \& Philosophy 7: 347-377.

Bartsch, R. and T. Vennemann. 1972. Semantic Structures: A Study in the Relation between Syntax and Semantics. Frankfurt: Athenaeum Verlag.

Bierwisch, M. 1989. The semantics of gradation. In Bierwisch, M. and E. Lang (eds.), Dimensional Adjectives. Berlin: Springer-Verlag. 71-262.

Cresswell, M.J. 1976. The semantics of degree. In B. Partee (ed.), Montague Grammar. New York: Academic Press. 261-292.

Gawron, J-M. 1995. Comparatives, superlatives, and resolution. Linguistics \& Philosophy 18:333-380.

Hale, A. 1970. Conditions on English comparative clause pairings. In Jacobs, R. and P. Rosenbaum (eds.) Readings in English Transformational Grammar. Washington: Georgetown University Press.

Hazout, I. 1995. Comparative ellipsis and Logical Form. Natural Language and Linguistic Theory 13:1-37.

Heim, I. 1985. Notes on comparatives and related matters. Ms., University of Texas, Austin.

Hellan, L. 1981. Towards an Integrated Analysis of Comparatives. Tübingen: Narr.

Hendriks, P. 1995. Comparatives and Categorial Grammar. Doctoral dissertation, University of Groningen.

Hoeksema, J. 1983. Negative polarity and the comparative. Natural Language and Linguistic Theory 1:403-434.

Kamp, H. 1975. Two theories of Adjectives. In Keenan, Edward. (ed.) Formal Semantics of Natural Language. Cambridge: Cambridge University Press, 123-155.

Kennedy, C. 1997. Projecting the Adjective: The Syntax and Semantics of Gradability and Comparison. Doctoral dissertation, University of California, Santa Cruz.

Kennedy, C. 1996. On the monotonicity of polar adjectives. Paper presented at the Perspectives on Negation workshop, 24-26 August, Rijksuniversiteit Groningen, the Netherlands.

Klein, E. 1991. Comparatives. In von Stechow, A. \& D. Wunderlich (eds.) Semantik: Ein internationales Handbuch der zeitgenössischen Forschung. Berlin: Walter de Gruyter.

Klein, E. 1980. A semantics for positive and comparative adjectives. Linguistics \& Philosophy 4.1:1-45.

Landman, F. 1991. Structures for Semantics. Dordrecht: Kluwer.

Larson, R. 1988. Scope and comparatives. Linguistics \& Philosophy 11:1-26.

Lerner, J-Y. and M. Pinkal. 1995. Comparative ellipsis and variable binding. Computerlinguistik an der Universität des Saarlandes Report No. 64. Also in The Proceedings of SALT 5.

Lerner, J-Y. and M. Pinkal. 1992. Comparatives and nested quantification. Computerlinguistik an der Universität des Saarlandes Report No. 21. 
Löbner, S. 1990. Wahr neben Falsch: Duale Operatoren als die Quantoren natürlicher Sprache. Tübingen: Niemeyer.

McConnell-Ginet, S. 1973. Comparative Constructions in English: A Syntactic and Semantic Analysis. Doctoral dissertation, University of Rochester.

Moltmann, F. 1992. Coordination and Comparatives. Ph.D. dissertation, MIT.

Postal, P. 1974. On certain ambiguities. Linguistic Inquiry 5:367-425.

Rullmann, H. 1995. Maximality in the Semantics of WH-Constructions. Ph.D. dissertation, UMass., Amherst.

Russell, B. 1905. On denoting. Mind 14:479-493.

Sánchez-Valencia, V. 1994. Polarity, predicates, and monotonicity. Ms., University of Groningen.

Seuren, P.A.M. 1984. The comparative revisited. Journal of Semantics 3:109-141.

Seuren, P.A.M. 1978. The structure and selection of positive and negative gradable adjectives. In Farkas, D., W. Jacobson \& K Todrys (eds.), Papers from the Parasession on the Lexicon, CLS 14, University of Chicago. 336-346.

Seuren, P.A.M. 1973. The comparative. In F. Kiefer \& N. Ruwet (eds.), Generative Grammar in Europe. Dordrecht: Riedel.

von Stechow, A. 1984a. Comparing semantic theories of comparison. Journal of Semantics 3:1-77.

von Stechow, A. 1984b. My reaction to Cresswell's, Hellan's, Hoeksema's, and Seuren's comments. Journal of Semantics 3:183-199.

Williams, E. 1977. Discourse and logical form. Linguistic Inquiry 8.1:101-139.

Wunderlich, D. 1970. Vergleischssätze. Ms., Berlin Technische Universität. 